\title{
Factors Associated with Patient Satisfaction to Healthcare at Mpigi Health Centre IV, Mpigi District-Uganda
}

\author{
Kizito Omona $[\mathrm{PhD}]^{1 *}$; Leticia Nakandi ${ }^{1}$; Annet Beatrice Nambi ${ }^{1}$; Fatuma Mayanja Nakayiza ${ }^{1}$; \\ Margaret Nanozi ${ }^{1}$; Mary Matilda Namuli ${ }^{1}$; Richard Zimbe ${ }^{1}$ \\ ${ }^{1}$ Faculty of Health Sciences, Uganda Martyrs University, Kampala, Uganda \\ Corresponding Author: Dr. Omona Kizito, Lecturer. E-mail: komona@umu.ac.ug, Tel: +256706464873 \\ DOI: 10.47760/cognizance.2021.v01i04.001
}

\begin{abstract}
Introduction: Patient satisfaction is important for measuring health service delivery, utilization and thus improving quality of care provided. Worldwide, patients are increasingly dissatisfied with the commercialization of medical services, bureaucratic healthcare system, poor quality of healthcare and rotting patient-healthcare provider relationships. Satisfaction is an expression of the gap between the expected and perceived characteristics of service.
\end{abstract}

Objective: The purpose of study was to establish factors associated with patient satisfaction to Healthcare at Mpigi Health Centre IV, Mpigi District- Uganda.

Materials \& Methods: An analytical cross-sectional study designs utilizing a quantitative approach was employed. 295 patients were selected using systematic random sampling and asked to complete a structured patient's exist-questionnaire. Data collected was coded and entered into the computer using SPSS version 22 for analysis. Univariate, bivariate and multivariate analysis was carried out. A multivariate Logistic regression model was used to determine the independent factors associated patients' satisfaction. Variables with p-value of $<0.05$ at $95 \%$ CI were used to declare statistical significance.

Results: Out of the 295 respondents data analysed, patients satisfaction was at 58.0\%. Most of the patients believed that services were just fair (39.0\%) and most of the patients (53.9\%) didn't believe that the staffs were responsive to patients' needs. Age of the respondents $(p=0.009)$, gender $(p=0.048)$, employment status of respondents $(p=0.005)$ were all found to be associated with patients' satisfaction. The study also found that staffs giving enough time and attention to explain patient problems $(p=0.005)$, according privacy during diagnosis and treatment $(p=0.019)$ and availability of medicine and supplies $(p=0.001)$ were the only health facility factors that facilitated patients' satisfaction.

Conclusions: Although this study revealed a fair level of patient satisfaction, certain aspects as per the results need to be addressed in order to enhance satisfaction. The study recommends strengthening of supervision, monitoring and evaluation; availing enough facilities and utilities; more satisfaction surveys; timely requisition of resources; training of staff in customer care, quality improvement and communication; reducing patient waiting time; availing a suggestion box; improved patient privacy and provision of adequate information to the patients.

Keywords: Patient satisfaction, Healthcare, Barriers, Facilitators, Quality of Care 


\section{INTRODUCTION \\ 1.1 Background of the study}

Patient satisfaction is a measure of the extent to which a patient is content with the health care provided and their experience while using a health service (1). It is an important measure of healthcare quality, health system performance and responsiveness and the extent to which health care meets patients' expectations $(2,3)$. Measuring patient satisfaction has become an integral part of hospital management strategies for quality assurance and accreditation process in most countries (4). Quality of care is the extent to which health care services provided to populations improve desired health outcomes and to achieve that, health care must be safe, effective, timely, efficient, equitable and people-centered (5).

Worldwide, patients are increasingly getting dissatisfied with the commercialization of medical services, bureaucratic healthcare system, poor quality of healthcare and rotting patient- healthcare provider relationships $(6,7)$. There is a high variation of patients' level of satisfaction between countries and within a country. In developed countries, patients are highly satisfied (90-95\%) with the health care provided while in developing countries, it has been shown that the range of patients' satisfaction varies between $95 \%$ to $<20 \%(9,2)$.

In Uganda, Studies conducted show a range of $40 \%-84.2 \%$ rates of client satisfaction (10), leaving many patients dissatisfied. Common reports from patients and adverse mass media reports have attributed unsatisfactory services to staff hostility and negligence, staff mistreatment of patients, gender discrimination, drug shortages, inadequate number of staffs and their absenteeism, among others (11).

Despite the need for continuous quality improvement, limited studies $(7,10,12)$ have been conducted on patient satisfaction with the Uganda public health sector and almost none particularly in health centre IVs. Even then, most of them were restricted to measure satisfaction for particular health services or programs.

If patients are dissatisfied with the quality of care, they may decide to seek treatment somewhere else outside the formal health system which may be detrimental to their health. Dissatisfaction can also lead to poor uptake of health services, poor adherence to medical care, treatment plans and recommendations, poor retention to services, inconsistent relationship with specific providers and at the end this may contribute to high morbidity and mortality $(13,14)$.

The Ministry of health in Uganda has implemented strategies to improve health care accessibility and quality through decentralization, Health Sector Strategic Plans, Comprehensive Health Policy, Quality Improvement Initiatives and Result Based Financing, among others. Despite these measures, dissatisfaction could be the reason why some patients do not use the available services. Thus, this study aims at exploring the barriers and facilitators of patient satisfaction so that findings can be used in designing and implementing service improvement plans.

\subsection{Broad Objective}

The purpose of study was to establish factors associated with patient satisfaction to Healthcare at Mpigi Health Centre IV, Mpigi District- Uganda. 




\subsection{Specific objectives}

(1) To determine the Level of patients' satisfaction with healthcare at Mpigi Health Centre IV

(2) To examine patients' perceptions on quality of care received at Mpigi Health Centre IV

(3) To establish whether there was an association between patients' perceptions of quality of Care and patients' Satisfaction at Mpigi Health Centre IV

(4) To ascertain the socio-demographic factors associated with patients' satisfaction at Mpigi Health Centre IV

(5) To examine the health facility factors associated with patients' satisfaction at Mpigi Health Centre IV

\section{MATERIALS AND METHODs}

\subsection{Study design}

The study was an analytical cross sectional study design that employed quantitative methods of data collection

\subsection{Study population}

The study population for this research was all patients present at Mpigi health Centre at the time of data collection.

\subsection{Inclusion and Exclusion Criteria}

The inclusion criteria were clients who got healthcare service(s) from the health facility and are exiting the facility. The exclusion criterion was any client below the age of consent (18 years) who is not accompanied by an adult and patients who did not speak Luganda or English.

\section{4 Sample size estimation}

The sample size was estimated using the Kish and Leisley formula of 1965 for cross sectional studies, $\boldsymbol{n}=\frac{Z^{2} p q}{d^{2}}$

Thus, $\boldsymbol{n}=295$ respondents

Where;

$$
\boldsymbol{n}=\text { the sample size }
$$

\subsection{Sampling procedure}

Participants were drawn from patients exiting the facility. Systematic random sampling was used to select study participants at their time of exist. One research assistant was assigned to recruit participants, selecting every $4^{\text {th }}$ client exiting the facility.

\subsection{Data collection}

A structured questionnaire was administered to patients exiting the healthcare facility to establish factors associated with their satisfaction. The tool was pre-tested in a different facility and modified accordingly. All patient interviews were conducted by the Principle investigator (PI). 


\subsection{Data Analysis}

This was done at all levels using SPSS version 22. Univariate analysis obtained the frequency and distribution of the study variables. Bivariate analysis used Chi-square test to determine the association between two categorical variables. Probability values $<0.05$ were considered significant. Multivariate analysis was performed to control the confounding variables by logistic regression analysis. Variables with $\mathrm{p}>0.05$ but $<0.1$ at bivariate analysis were put into the model to establish factors independently associated with patient satisfaction.

\subsection{Ethical considerations}

The researchers obtained an approval from the research ethics committee at Uganda Martyrs University. Participants were voluntarily asked to take part in the study and an informed consent was sought prior to interviews.

\section{RESULTS}

\subsection{Socio-demographic Characteristics of Respondents}

The socio-demographic characteristics of patients are shown in table 1 below. Most of the respondents were aged 25-34 years accounting 39.0\% and majority 176(59.7\%) were female. Most of the respondents $(135(45.8 \%))$ were married.

Table 1: Socio-demographic Characteristics of the Respondents

\begin{tabular}{|c|c|c|}
\hline Variables & Frequency & Percentage (\%) \\
\hline $\begin{array}{l}\text { Age } \\
: \quad 18-24 \text { years } \\
: \quad 25-34 \text { years } \\
: \quad 35-44 \text { years } \\
: 45 \text { years }\end{array}$ & $\begin{array}{l}68 \\
115 \\
69 \\
43\end{array}$ & $\begin{array}{l}23.1 \\
39.0 \\
23.4 \\
14.6\end{array}$ \\
\hline $\begin{aligned} \text { Gender } & \\
\text { - } & \text { Male } \\
\text { - } & \text { Female }\end{aligned}$ & $\begin{array}{l}119 \\
176 \\
\end{array}$ & $\begin{array}{l}40.3 \\
59.7 \\
\end{array}$ \\
\hline $\begin{aligned} & \text { Education level } \\
& \text { - } \text { None } \\
& \text { : } \text { Primary } \\
& \text { - } \text { Tecondary } \\
&\end{aligned}$ & $\begin{array}{l}59 \\
99 \\
110 \\
27\end{array}$ & $\begin{array}{l}20.0 \\
33.6 \\
37.3 \\
9.2\end{array}$ \\
\hline $\begin{aligned} \text { Income per Month (in Ug. Shs) } \\
\text { : } \quad \text { UGX }<100,000 /= \\
: \quad \text { UGX100,000/= - UGX500,000/= } \\
\text { : } \quad \text { UGX500,001/= - UGX1,000,000/= }>1,000,000 /=\end{aligned}$ & $\begin{array}{l}91 \\
126 \\
57 \\
21\end{array}$ & $\begin{array}{l}30.8 \\
42.7 \\
19.3 \\
7.1\end{array}$ \\
\hline $\begin{array}{cl}\text { Employment status } \\
: & \text { Employed } \\
\text { - } & \text { Self-employed } \\
\text { - Unemployed }\end{array}$ & $\begin{array}{l}78 \\
105 \\
112\end{array}$ & $\begin{array}{l}26.4 \\
35.6 \\
38.0\end{array}$ \\
\hline $\begin{aligned} & \text { Marital status } \\
&: \text { Single } \\
& \text { : } \text { Married } \\
& \text { : } \text { Divorced } \\
& \text { Widow/widower }\end{aligned}$ & $\begin{array}{l}84 \\
135 \\
42 \\
34\end{array}$ & $\begin{array}{l}28.5 \\
45.8 \\
14.2 \\
11.5\end{array}$ \\
\hline
\end{tabular}

Source: Primary, $\boldsymbol{U G X}=$ Ugandan Shillings, Shs $=$ Shillings 
cognizancejournal.com

Kizito Omona et al, Cognizance Journal of Multidisciplinary Studies, Vol.1, Issue.4, April 2021, pg. 1-12

\subsection{Level of Patients' Satisfaction with Healthcare}

Out of the 295 respondents data analyzed, patients satisfaction was at $58 \%$, amounting to only 172 patients. Henceforth, $42 \%$ of the respondents were dissatisfied with the healthcare.

\subsection{Patients' Perceptions on Quality of Care Received}

The respondents were asked to rate their feelings on quality of care they received. Rating was done on a likert scale with a number of variables as shown in table 2 below

Table 2: Patients Perceptions on Quality of Care

\begin{tabular}{|c|c|c|}
\hline Variables & Frequency & Percentage $(\%)$ \\
\hline $\begin{array}{l}\text { Physical environment is appealing } \\
\text { - Agree } \\
\text { - Disagree }\end{array}$ & $\begin{array}{l}43 \\
252 \\
\end{array}$ & $\begin{array}{r}14.6 \\
85.4 \\
\end{array}$ \\
\hline $\begin{array}{l}\text { There is adequate seating area } \\
\text { - Agree } \\
\text { - Disagree }\end{array}$ & $\begin{array}{l}154 \\
141\end{array}$ & $\begin{array}{l}52.2 \\
47.8 \\
\end{array}$ \\
\hline $\begin{array}{l}\text { Staff provided services on time } \\
\text { - Agree } \\
\text { - } \text { Disagree }\end{array}$ & $\begin{array}{l}112 \\
183\end{array}$ & $\begin{array}{l}38.0 \\
62.0\end{array}$ \\
\hline $\begin{array}{l}\text { Consistency in performance by staff } \\
\text { - Agree } \\
\text { - Disagree }\end{array}$ & $\begin{array}{l}78 \\
217\end{array}$ & $\begin{array}{l}26.4 \\
73.6\end{array}$ \\
\hline $\begin{array}{l}\text { Staff were helpful } \\
\text { - Agree } \\
\text { - Disagree }\end{array}$ & $\begin{array}{l}183 \\
112\end{array}$ & $\begin{array}{l}62.0 \\
38.0\end{array}$ \\
\hline $\begin{array}{l}\text { Staff were responsive to patient needs } \\
\text { - Agree } \\
\text { - Disagree }\end{array}$ & $\begin{array}{l}136 \\
159\end{array}$ & $\begin{array}{l}46.1 \\
53.9\end{array}$ \\
\hline $\begin{array}{l}\text { Staff responded to me immediately when called } \\
\text { - Agree } \\
\text { - Disagree }\end{array}$ & $\begin{array}{l}138 \\
157 \\
\end{array}$ & $\begin{array}{l}46.8 \\
53.2 \\
\end{array}$ \\
\hline $\begin{array}{l}\text { Prompt service delivery } \\
\text { - Agree } \\
\text { - Disagree } \\
\end{array}$ & $\begin{array}{l}81 \\
214\end{array}$ & $\begin{array}{l}27.5 \\
72.5\end{array}$ \\
\hline $\begin{array}{l}\text { Staff treat patients with dignity and respect } \\
\text { - Agree } \\
\text { - Disagree }\end{array}$ & $\begin{array}{l}108 \\
187\end{array}$ & $\begin{array}{l}36.6 \\
63.4\end{array}$ \\
\hline $\begin{array}{l}\text { Staff understood my specific needs } \\
\text { - Agree } \\
\text { - } \quad \text { Disagree }\end{array}$ & $\begin{array}{l}88 \\
207\end{array}$ & $\begin{array}{l}29.8 \\
70.2\end{array}$ \\
\hline $\begin{array}{l}\text { Staff gave me special attention } \\
\text { - Agree } \\
\text { - Disagree }\end{array}$ & $\begin{array}{l}88 \\
207\end{array}$ & $\begin{array}{l}29.8 \\
70.2\end{array}$ \\
\hline $\begin{array}{l}\text { Staff were caring to patients } \\
\text { - Agree } \\
\text { - Disagree }\end{array}$ & $\begin{array}{l}101 \\
194 \\
\end{array}$ & $\begin{array}{l}34.2 \\
65.8 \\
\end{array}$ \\
\hline
\end{tabular}




\begin{tabular}{|c|c|c|}
\hline $\begin{array}{l}\text { I received adequate explanation of tests I had to undergo } \\
\text { - Agree } \\
\text { - Disagree }\end{array}$ & $\begin{array}{l}84 \\
211\end{array}$ & $\begin{array}{l}28.5 \\
71.5\end{array}$ \\
\hline $\begin{array}{l}\text { Doctors were willing to answer any questions relating to } \\
\text { illness. } \\
\text { - Agree } \\
\text { - Disagree }\end{array}$ & $\begin{array}{l}222 \\
73\end{array}$ & $\begin{array}{l}75.3 \\
24.7\end{array}$ \\
\hline $\begin{array}{l}\text { I was given adequate information on my health condition. } \\
\text { - Agree } \\
\text { - Disagree }\end{array}$ & $\begin{array}{l}213 \\
82\end{array}$ & $\begin{array}{l}72.2 \\
27.8\end{array}$ \\
\hline $\begin{array}{l}\text { I was given adequate information on my treatment } \\
\text { - Agree } \\
\text { - Disagree }\end{array}$ & $\begin{array}{l}187 \\
108\end{array}$ & $\begin{array}{l}63.4 \\
36.6\end{array}$ \\
\hline $\begin{array}{l}\text { The facility is accessible } \\
\text { - Agree } \\
\text { - Disagree }\end{array}$ & $\begin{array}{l}213 \\
82\end{array}$ & $\begin{array}{l}72.2 \\
27.8\end{array}$ \\
\hline $\begin{array}{l}\text { I was charged for services } \\
\text { - Agree } \\
\text { - Disagree }\end{array}$ & $\begin{array}{l}143 \\
152\end{array}$ & $\begin{array}{l}48.5 \\
51.5\end{array}$ \\
\hline $\begin{array}{l}\text { Rating the services provided at the facility } \\
\text { - Excellent } \\
\text { - Very good } \\
\text { - Good } \\
\text { - Fair } \\
\text { - Poor }\end{array}$ & $\begin{array}{l}5 \\
70 \\
85 \\
115 \\
20 \\
\end{array}$ & $\begin{array}{l}1.7 \\
23.7 \\
28.8 \\
39.0 \\
6.8\end{array}$ \\
\hline
\end{tabular}

Source: Primary

Very few patients $(1.7 \%)$ believed that services were excellent and very few $(6.8 \%)$ believed that services were that poor. However, most of the patients believed that services were only fair, accounting for 39.0\% of the respondents. Most of the patients $(53.9 \%)$ didn't believe that Staffs were responsive to patients' needs.

\subsection{Association Between Patients' Perception of Quality of Care and Satisfaction}

A bivariate analysis was done to establish whether there was association between patients' perception of quality of care and their satisfaction. Results are shown in table 3 below

Table 3: Patients Perception on quality associated with patient satisfaction

\begin{tabular}{|c|c|c|c|c|}
\hline \multirow[t]{2}{*}{ Variables } & \multicolumn{2}{|c|}{ Satisfied } & \multirow[t]{2}{*}{$\chi^{2}$} & \multirow{2}{*}{$\begin{array}{l}\text { p- } \\
\text { value }\end{array}$} \\
\hline & Yes $(\%)$ & No $(\%)$ & & \\
\hline $\begin{array}{l}\text { Physical environment is appealing } \\
\text { - Agree } \\
\text { - Disagree }\end{array}$ & $\begin{array}{l}21(12.3 \%) \\
150(87.7 \%)\end{array}$ & $\begin{array}{l}22(17.7 \%) \\
102(82.3 \%)\end{array}$ & $\begin{array}{l}1.722 \\
\mathrm{Df}=1\end{array}$ & .189 \\
\hline $\begin{array}{l}\text { There are adequate seats } \\
\text { - Agree } \\
\text { - Disagree }\end{array}$ & $\begin{array}{l}89(52.0 \%) \\
82(48.0 \%)\end{array}$ & $\begin{array}{l}65(52.4 \%) \\
59(47.6 \%)\end{array}$ & $\begin{array}{l}11.950 \\
\mathrm{Df}=1\end{array}$ & $.004 *$ \\
\hline $\begin{array}{l}\text { Staff provide services on time } \\
\text { - Agree } \\
\text { - } \text { Disagree }\end{array}$ & $\begin{array}{l}64(37.4 \%) \\
107(62.6 \%)\end{array}$ & $\begin{array}{l}107(62.6 \%) \\
76(61.3 \%)\end{array}$ & $\begin{array}{l}.823 \\
\mathrm{Df}=1\end{array}$ & .050 \\
\hline $\begin{array}{l}\text { Consistency in performance by staff } \\
\text { - Agree } \\
\text { - Disagree }\end{array}$ & $\begin{array}{l}49(28.7 \%) \\
122(71.3 \%)\end{array}$ & $\begin{array}{l}29(23.4 \%) \\
95(76.6 \%)\end{array}$ & $\begin{array}{l}1.026 \\
\mathrm{Df}=1\end{array}$ & .426 \\
\hline
\end{tabular}


cognizancejournal.com

Kizito Omona et al, Cognizance Journal of Multidisciplinary Studies, Vol.1, Issue.4, April 2021, pg. 1-12

\begin{tabular}{|c|c|c|c|c|}
\hline $\begin{array}{l}\text { Staff was helpful to patients/ me } \\
\text { - Agree } \\
\text { - } \text { Disagree }\end{array}$ & $\begin{array}{l}99(57.9 \%) \\
72(42.1 \%) \\
\end{array}$ & $\begin{array}{l}84(67.7 \%) \\
40(32.3 \%) \\
\end{array}$ & $\begin{array}{l}2.959 \\
\mathrm{Df}=1\end{array}$ & .085 \\
\hline $\begin{array}{l}\text { Staff was responsive to patient needs } \\
\text { - Agree } \\
\text { - } \quad \text { Disagree }\end{array}$ & $\begin{array}{l}83(48.5 \%) \\
88(51.5 \%)\end{array}$ & $\begin{array}{l}53(42.7 \%) \\
71(57.3 \%) \\
\end{array}$ & $\begin{array}{l}.972 \\
\mathrm{Df}=1\end{array}$ & .324 \\
\hline $\begin{array}{l}\text { Staff responded immediately when called by the patients } \\
\text { - Agree } \\
\text { - Disagree }\end{array}$ & $\begin{array}{l}94(55.0 \%) \\
77(45.0 \%) \\
\end{array}$ & $\begin{array}{l}44(35.5 \%) \\
80(64.5 \%) \\
\end{array}$ & $\begin{array}{l}10.963 \\
\mathrm{Df}=1\end{array}$ & $.001 *$ \\
\hline $\begin{array}{l}\text { Prompt service delivery } \\
\text { - Agree } \\
\text { - } \quad \text { Disagree } \\
\end{array}$ & $\begin{array}{l}57(33.3 \%) \\
114(66.7 \%)\end{array}$ & $\begin{array}{l}24(19.4 \%) \\
100(80.6 \%)\end{array}$ & $\begin{array}{l}7.051 \\
\mathrm{Df}=1\end{array}$ & $.008 *$ \\
\hline $\begin{array}{l}\text { Staff treat patients with dignity and respect } \\
\text { - Agree } \\
\text { - } \text { Disagree }\end{array}$ & $\begin{array}{l}64(37.4 \%) \\
107(62.6 \%)\end{array}$ & $\begin{array}{l}44(35.5 \%) \\
80(64.5 \%)\end{array}$ & $\begin{array}{l}.117 \\
\mathrm{Df}=1\end{array}$ & .732 \\
\hline $\begin{array}{l}\text { Staff understand my specific needs } \\
\text { - Agree } \\
\text { - } \quad \text { Disagree }\end{array}$ & $\begin{array}{l}51(29.8 \%) \\
120(70.2 \%)\end{array}$ & $\begin{array}{l}37(29.8 \%) \\
87(70.2 \%)\end{array}$ & $\begin{array}{l}10.998 \\
\text { Df }=1\end{array}$ & $.000 *$ \\
\hline $\begin{array}{l}\text { Staff gave me special attention } \\
\text { - Agree } \\
\text { - } \quad \text { Disagree }\end{array}$ & $\begin{array}{l}56(32.7 \%) \\
115(67.3 \%)\end{array}$ & $\begin{array}{l}32(25.8 \%) \\
92(74.2 \%) \\
\end{array}$ & $\begin{array}{l}1.655 \\
\mathrm{Df}=1\end{array}$ & .198 \\
\hline $\begin{array}{l}\text { Staff at the hospital were caring } \\
\text { - Agree } \\
\text { - Disagree }\end{array}$ & $\begin{array}{l}59(34.5 \%) \\
112(65.5 \%)\end{array}$ & $\begin{array}{l}42(33.9 \%) \\
82(66.1 \%) \\
\end{array}$ & $\begin{array}{l}\text { 8. } .910 \\
\mathrm{Df}=1\end{array}$ & $.013 *$ \\
\hline $\begin{array}{l}\text { I received adequate explanation of any tests I had to } \\
\text { undergo } \\
\text { - Agree } \\
\text { - Disagree }\end{array}$ & $\begin{array}{l}51(29.8 \%) \\
120(70.2 \%)\end{array}$ & $\begin{array}{l}33(26.6 \%) \\
91(73.4 \%) \\
\end{array}$ & $\begin{array}{l}.364 \\
\text { Df }=1\end{array}$ & .546 \\
\hline $\begin{array}{l}\text { The doctors were willing to answer any questions } \\
\text { - Agree } \\
\text { - Disagree }\end{array}$ & $\begin{array}{l}126(73.7 \%) \\
45(26.3 \%)\end{array}$ & $\begin{array}{l}96(77.4 \%) \\
28(22.6 \%) \\
\end{array}$ & $\begin{array}{l}43.538 \\
\mathrm{Df}=1\end{array}$ & $.003 *$ \\
\hline $\begin{array}{l}\text { I was given adequate information on my health condition } \\
\text { - Agree } \\
\text { - Disagree }\end{array}$ & $\begin{array}{l}126(73.7 \%) \\
45(26.3 \%)\end{array}$ & $\begin{array}{l}87(70.2 \%) \\
37(29.8 \%) \\
\end{array}$ & $\begin{array}{l}.444 \\
\mathrm{Df}=1\end{array}$ & .505 \\
\hline $\begin{array}{l}\text { I was given adequate information on my treatment } \\
\text { - Agree } \\
\text { - Disagree }\end{array}$ & $\begin{array}{l}106(62.0 \%) \\
65(38.0 \%)\end{array}$ & $\begin{array}{l}81(65.3 \%) \\
43(34.7 \%)\end{array}$ & $\begin{array}{l}15.344 \\
\mathrm{Df}=1\end{array}$ & $.007 *$ \\
\hline $\begin{array}{l}\text { The facility is accessible } \\
\text { - Agree } \\
\text { - Disagree }\end{array}$ & $\begin{array}{l}119(69.6 \%) \\
52(30.4 \%)\end{array}$ & $\begin{array}{l}94(75.8 \%) \\
30(24.2 \%) \\
\end{array}$ & $\begin{array}{l}1.384 \\
\mathrm{Df}=1\end{array}$ & .239 \\
\hline $\begin{array}{l}\text { I was charged for services } \\
\text { - Agree } \\
\text { - } \quad \text { Disagree }\end{array}$ & $\begin{array}{l}71(41.5 \%) \\
100(58.5 \%)\end{array}$ & $\begin{array}{l}72(58.1 \%) \\
52(41.9 \%) \\
\end{array}$ & $\begin{array}{l}7.877 \\
\mathrm{Df}=1\end{array}$ & $.005 *$ \\
\hline $\begin{array}{l}\text { Rating the services provided } \\
\text { - Excellent } \\
\text { - Very good } \\
\text { - Good } \\
\text { - Fair } \\
\text { - Poor }\end{array}$ & $\begin{array}{l}3(1.8 \%) \\
49(28.7 \%) \\
48(28.1 \%) \\
56(32.7 \%) \\
15(8.8 \%)\end{array}$ & $\begin{array}{l}2(1.6 \%) \\
21(16.9 \%) \\
37(29.8 \%) \\
59(47.6 \%) \\
5(4.0 \%)\end{array}$ & $\begin{array}{l}13.78 \\
\mathrm{Df}=4\end{array}$ & $.036 *$ \\
\hline
\end{tabular}

Source: Primary, $\quad *<0.05$ significance, $\chi^{2}=$ Chi-square test, $\boldsymbol{D} \boldsymbol{f}=$ Degree of freedom

The study found that perception that there was adequate seat $(\mathrm{p}=0.004)$, staff responded immediately when called by the patients $(p=0.001)$, prompt service delivery $(p=0.008)$, Staff understand my specific needs $(\mathrm{p}=0.000)$, staff at the hospital were caring $(\mathrm{p}=0.013)$, doctors were willing to answer any questions $(\mathrm{p}=0.003)$, being given adequate information on treatment $(\mathrm{p}=0.007)$ and rating services $(\mathrm{p}=0.036)$ were associated with patients satisfaction. 
cognizancejournal.com

Kizito Omona et al, Cognizance Journal of Multidisciplinary Studies, Vol.1, Issue.4, April 2021, pg. 1-12

\subsection{Socio-demographic Factors Associated with Patients' Satisfaction}

A bivariate analysis was done to establish the socio-demographic factors associated with patients' satisfaction. Results are shown in table 4 below

Table 4: Socio-demographic characteristics associated with patients' satisfaction

\begin{tabular}{|c|c|c|c|c|}
\hline \multirow[t]{2}{*}{ Variables } & \multicolumn{2}{|c|}{ Satisfied } & \multirow[t]{2}{*}{$\chi^{2}$} & \multirow[t]{2}{*}{ p-value } \\
\hline & Yes (\%) & No $(\%)$ & & \\
\hline $\begin{array}{ll}\text { Age } & \\
\text { - } & 18-24 \text { years } \\
\text { - } & 25-34 \text { years } \\
\text { - } & 35-44 \text { years } \\
\text { - } & \geq 45 \text { years }\end{array}$ & $\begin{array}{l}44(25.7 \%) \\
66(38.6 \%) \\
38(22.2 \%) \\
23(13.5 \%)\end{array}$ & $\begin{array}{l}24(19.4 \%) \\
49(39.5 \%) \\
31(25.0 \%) \\
20(16.1 \%)\end{array}$ & $\begin{array}{l}15.874 \\
\mathrm{Df}=3\end{array}$ & $.009 *$ \\
\hline $\begin{aligned} & \text { Gender } \\
& \text { - } \text { Male } \\
& \text { - } \text { Female } \\
&\end{aligned}$ & $\begin{array}{l}72(42.1 \%) \\
99(57.9 \%) \\
\end{array}$ & $\begin{array}{l}47(37.9 \%) \\
77(62.1 \%)\end{array}$ & $\begin{array}{l}8.527 \\
\mathrm{Df}=1\end{array}$ & $.048 *$ \\
\hline $\begin{array}{l}\text { Education level } \\
\text { - } \text { None } \\
\text { - Primary } \\
\text { - Secondary } \\
\text { - Tertiary }\end{array}$ & $\begin{array}{l}34(19.9 \%) \\
52(30.4 \%) \\
70(40.9 \%) \\
15(8.8 \%)\end{array}$ & $\begin{array}{l}25(20.2 \%) \\
47(37.9 \%) \\
40(32.3 \%) \\
12(9.7 \%)\end{array}$ & $\begin{array}{l}2.722 \\
\mathrm{Df}=3\end{array}$ & .437 \\
\hline $\begin{array}{cl}\text { Income per Month } \\
\text { - } \mathrm{UGX}<100,000 /= \\
\text { - } \mathrm{UGX} 100,000 /=-\mathrm{UGX} 500,000 /= \\
\text { - } \mathrm{UGX} 500,001-1,000,000 /= \\
\text { - } \mathrm{UGX}>1,000,000 /=\end{array}$ & $\begin{array}{l}54(31.6 \%) \\
66(38.6 \%) \\
38(22.2 \%) \\
13(7.6 \%)\end{array}$ & $\begin{array}{l}37(29.8 \%) \\
60(48.4 \%) \\
19(15.3 \%) \\
8(6.5 \%)\end{array}$ & $\begin{array}{l}3.588 \\
\mathrm{Df}=3\end{array}$ & .309 \\
\hline $\begin{array}{cl}\text { Employment status } \\
\text { - } & \text { Employed } \\
\text { - } & \text { Self-employed } \\
\text { - } & \text { Unemployed } \\
\end{array}$ & $\begin{array}{l}40(23.4 \%) \\
61(35.7 \%) \\
70(40.9 \%) \\
\end{array}$ & $\begin{array}{l}38(30.6 \%) \\
44(35.5 \%) \\
42(33.9 \%)\end{array}$ & $\begin{array}{l}23.376 \\
\mathrm{Df}=2\end{array}$ & $.005^{*}$ \\
\hline $\begin{aligned} & \text { Marital status } \\
& \text { - } \text { Single } \\
& \text { - } \text { Married } \\
& \text { - } \text { Divorced } \\
& \text { - } \text { Widow/widower }\end{aligned}$ & $\begin{array}{l}44(25.7 \%) \\
79(46.2 \%) \\
23(13.5 \%) \\
25(14.6 \%)\end{array}$ & $\begin{array}{l}40(32.3 \%) \\
56(45.2 \%) \\
19(15.3 \%) \\
9(7.3 \%)\end{array}$ & $\begin{array}{l}4.649 \\
\mathrm{Df}=3\end{array}$ & .199 \\
\hline
\end{tabular}

Source: Primary, $*<0.05$ significance, $\chi^{2}-$ Chi-square test, $\boldsymbol{D} \boldsymbol{f}=$ Degree of freedom, $\boldsymbol{U} \boldsymbol{G} \boldsymbol{X}=$ Ugandan Shillings

Age of the respondents $(\mathrm{p}=0.009)$, gender $(\mathrm{p}=0.048)$, employment status of respondents $(\mathrm{p}=0.005)$ were all found to be associated with patients' satisfaction.

\subsection{Health Facility Factors Associated with Patients' Satisfaction}

To establish the health facility factors associated with patients' satisfaction, a bivariate analysis was done. Results are shown in table 5 below

Table 5: Health Facility Facilitators of Patients'Satisfaction

\begin{tabular}{|c|c|c|c|c|}
\hline \multirow[t]{2}{*}{ Variables } & \multicolumn{2}{|c|}{ Satisfied } & \multirow[t]{2}{*}{$\chi^{2}$} & \multirow{2}{*}{$\begin{array}{l}\text { p- } \\
\text { value }\end{array}$} \\
\hline & Yes $(\%)$ & No $(\%)$ & & \\
\hline $\begin{array}{l}\text { Availability of health workers } \\
\text { - Yes } \\
\text { - No }\end{array}$ & $\begin{array}{l}51(29.8 \%) \\
120(70.2 \%)\end{array}$ & $\begin{array}{l}35(28.2 \%) \\
89(71.8 \%)\end{array}$ & $\begin{array}{l}.089 \\
\mathrm{Df}=1\end{array}$ & .766 \\
\hline $\begin{array}{l}\text { Staff give enough time and attention to explain problem } \\
\text { - Yes }\end{array}$ & $87(50.9 \%)$ & $61(49.2 \%)$ & 27.082 & $.005 *$ \\
\hline
\end{tabular}


cognizancejournal.com

Kizito Omona et al, Cognizance Journal of Multidisciplinary Studies, Vol.1, Issue.4, April 2021, pg. 1-12

\begin{tabular}{|c|c|c|c|c|}
\hline - $\quad$ No & $84(49.1 \%)$ & $63(50.8 \%)$ & $\mathrm{Df}=1$ & \\
\hline $\begin{array}{l}\text { Convinced with the Health workers explanation } \\
\text { - Yes } \\
\text { - No }\end{array}$ & $\begin{array}{l}99(57.9 \%) \\
72(42.1 \%)\end{array}$ & $\begin{array}{l}61(49.2 \%) \\
63(50.8 \%) \\
\end{array}$ & $\begin{array}{l}2.193 \\
\mathrm{Df}=1\end{array}$ & .139 \\
\hline $\begin{array}{l}\text { Understood what the health worker explained } \\
\text { Y Yes } \\
\text { No }\end{array}$ & $\begin{array}{l}108(63.2 \%) \\
63(36.8 \%)\end{array}$ & $\begin{array}{l}88(71.0 \%) \\
36(29.0 \%)\end{array}$ & $\begin{array}{l}1.966 \\
\mathrm{Df}=1\end{array}$ & .161 \\
\hline $\begin{array}{l}\text { Privacy during diagnosis and treatment } \\
\text { - Yes } \\
\text { - No }\end{array}$ & $\begin{array}{l}98(57.3 \%) \\
73(42.7 \%)\end{array}$ & $\begin{array}{l}73(58.9 \%) \\
51(41.1 \%)\end{array}$ & $\begin{array}{l}17.072 \\
D f=1\end{array}$ & $.019 *$ \\
\hline $\begin{array}{l}\text { Facility operates all hours of the day } \\
\text { - Yes } \\
\text { - No }\end{array}$ & $\begin{array}{l}79(46.2 \%) \\
92(53.8 \%)\end{array}$ & $\begin{array}{l}63(50.8 \%) \\
61(49.2 \%)\end{array}$ & $\begin{array}{l}.611 \\
D f=1\end{array}$ & .434 \\
\hline $\begin{array}{l}\text { Clinics which didn't operate all hours of the day } \\
\text { - PNC/ANC } \\
\text { - } \text { OPD } \\
\text { - } \text { ART clinic } \\
\text { - Others } \\
\end{array}$ & $\begin{array}{l}9(9.8 \%) \\
48(52.2 \%) \\
13(14.1 \%) \\
18(19.6 \%) \\
4(4.3 \%) \\
\end{array}$ & $\begin{array}{l}13(21.3 \%) \\
26(42.6 \%) \\
14(23.0 \%) \\
7(11.5 \%) \\
1(1.6 \%)\end{array}$ & $\begin{array}{l}7.992 \\
\mathrm{Df}=4\end{array}$ & .092 \\
\hline $\begin{array}{l}\text { Educated about the patients' rights } \\
\text { : Yes } \\
\text { - No }\end{array}$ & $\begin{array}{l}67(39.2 \%) \\
104(60.8 \%)\end{array}$ & $\begin{array}{l}58(46.8 \%) \\
66(53.2 \%)\end{array}$ & $\begin{array}{l}1.697 \\
\mathrm{Df}=1\end{array}$ & 193 \\
\hline $\begin{array}{c}\text { Received the treatment you sought } \\
-\quad \text { Yes } \\
\text { - No }\end{array}$ & $\begin{array}{l}52(30.4 \%) \\
119(69.6 \%)\end{array}$ & $\begin{array}{l}43(34.7 \%) \\
81(65.3 \%) \\
\end{array}$ & $\begin{array}{l}.600 \\
\mathrm{Df}=1\end{array}$ & .439 \\
\hline $\begin{array}{l}\text { Medicines and supplies always available } \\
\text { - Yes } \\
\text { - No }\end{array}$ & $\begin{array}{l}59(34.5 \%) \\
112(65.5 \%)\end{array}$ & $\begin{array}{l}43(34.7 \%) \\
81(65.3 \%)\end{array}$ & $\begin{array}{l}13.795 \\
\mathrm{Df}=1\end{array}$ & $.001 *$ \\
\hline
\end{tabular}

Source: Primary, $\quad *<0.05$ significance, $\chi^{2}-$ Chi-square test, $\boldsymbol{D} \boldsymbol{f}=$ Degree of Freedom

The study found that Staff giving enough time and attention to explain patient problem $(\mathrm{p}=0.005)$, according privacy during diagnosis and treatment $(\mathrm{p}=0.019)$ and availability of medicine and supplies $(\mathrm{p}=0.001)$ were the only health facility factors that facilitated patients' satisfaction.

\section{DISCUSSIONS}

\subsection{Level of Patient Satisfaction}

The level of patient's satisfaction at Mpigi Health Center IV was found to be at $58.0 \%$. This falls within the range of 40-84.2\% in a Ugandan study on client satisfaction conducted (10). This is far lower compared to that of developed countries where satisfaction levels range between $90-95 \%(9 ; 2)$.

\subsection{Patient Perception of Quality of Care}

Basing on the likert scale (excellent, very good, good, fair and poor), majority of the participants rated quality of care as fair (39\%) and only $1.7 \%$ of the participants rated it excellent. Most of the participants disagreed to presence of the different dimensions of quality that this study assessed. These findings are somehow related to findings of a study that assessed the community perceptions on quality of health care delivery in two Uganda districts (using a scale of good and low) that revealed the quality to be low (15). Patients who perceived the quality as unsatisfactory are likely to be dissatisfied with the health care as well. This view is similar to those found in a recent study on determinant of health service utilization (16). 


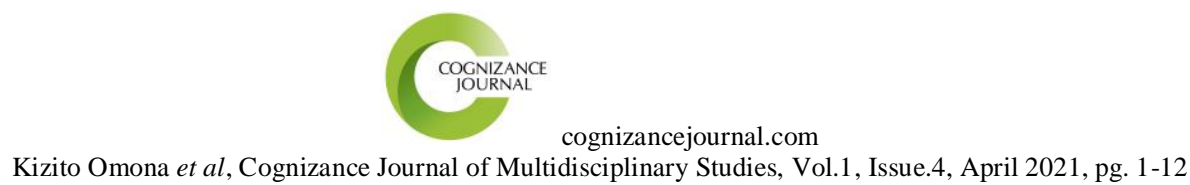

\subsection{Facilitators of Patient Satisfaction}

Age was found to have significant association with patients satisfaction; respondents who were at-least 45 years were 3.6 times more likely to be satisfied compared to 18-24 year olds. This is in agreement with a study which identified older age to be associated with higher satisfaction (17). This could be that from their long lived experiences, there have been subsequent better improvements in the overall health services making them grateful and easily appreciative.

The study also found that employment status was significantly associated with patient satisfaction with health services; respondents who were unemployed were 0.205 times less likely to be satisfied with the health services compared to those who were employed. This is in line with a study conducted in a mental hospital, which found that employment status has an influence on patient satisfaction; being employed and being able to work were associated with higher satisfaction (17). It is more likely that in the mental institution some services required payment and the employed were at great advantage of receiving them more so, being able to work gave the patients hope of recovery. However, contradicting findings were found in a study conducted in Ethiopia where being unemployed was significantly associated with patient satisfaction (18). The difference could be that the Ethiopian study is likely to have been conducted in a facility where almost all services are free and therefore the facility favoured more the unemployed to access services due to availability of time at their disposal.

However, willingness for doctors to respond to any questions about their illness and provision of adequate information about the treatment were significantly associated with patient satisfaction according to the study findings. This is in agreement with a study which found out that giving enough information about the illness and problem and availability of counseling services influenced patient satisfaction (19). Furthermore, one study (20) found out that when patients understand their problems it facilitates their satisfaction. This is also in agreement with findings of the meta analysis review were active and regular interpersonal communication, feedback mechanisms and confidentiality enhanced patient satisfaction (21). Therefore service providers should aim at improving effective communication in all their interaction with patients.

Availability of medicines and supplies was a promoter of satisfaction with services at the health centre. This is in line with in a study carried out in Ethiopia in which providing free health care and availability of medication was significantly associated with patient satisfaction (18). This is similar to a study conducted in Egypt where lack of prescribed drugs and laboratory investigations were the main complaints affecting patients' satisfaction (22). A global situation review through a meta review identified shortage of resources in health facilities among the barriers to quality of care in maternal, new-born and child health (20). This poor quality of care can lead to patients' dissatisfaction and poor outcome. Thus, it is important that governments provide adequate resources in public health facilities to enhance patients' satisfaction and consequently improve facility utilization.

Privacy during diagnosis and treatment was found to have a significant association with patients' satisfaction. This is supported by one (22) who found that Lack of privacy causes patients dissatisfaction. In a study conducted in Nigeria $33 \%$ of the patients described privacy in consultation rooms as unsatisfactory and as such affect the patients experience with health care. Service providers should provide maximum privacy to their patients to enhance satisfaction. 


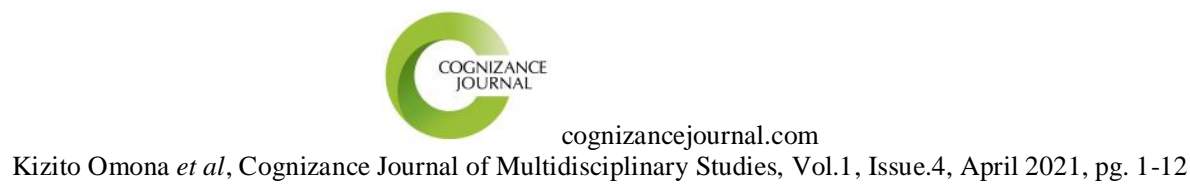

Patients who were promptly attended to were more satisfied with the services at the health facility according to this study. Patients reported waiting for health care staff at the facility for some time before being attended to as they started later than the opening time. This concurs with another scholar (23) who discovered that patients who were seen within 1 hour were 3.3 times more likely to be satisfied with their consultation. This is consistent with a study conducted at Mulago hospital which found out that a shorter waiting time was associated with increased satisfaction with intrapartum care (24). This further emphasizes the call for efforts of service providers to design and implement strategies geared at reducing patients waiting time.

\section{CONCLUSION}

The general patients' satisfaction level with healthcare at Mpigi Health centre IV was moderate (58.0\%). Most participants (39\%) perceived quality of healthcare as fair. Significant facilitator of patient satisfaction to health care included being employed, being above 45 years of age, receiving adequate information about treatment, availability of medicines and supplies, having privacy during diagnosis and treatment and willingness of doctors to answer illness related questions.

\section{RECOMMENDATIONS}

The authors therefore, recommend strengthening of supervision, monitoring and evaluation; availing enough facilities and utilities; more satisfaction surveys; timely requisition of resources; training of staff in customer care, quality improvement and communication; reducing patient waiting time; availing a suggestion box; improving patient privacy and provision of adequate information to the patients.

\section{ACKNOWLEDGEMENTS}

The authors would like to thank all the participants of this study and management of Mpigi Health Centre IV for their support which led to the successful completion of this study. We remain indebted to the staffs of Uganda Martyrs University, Faculty of Health Sciences.

\section{DECLARATION}

The authors declare that they had no conflict of interest.

\section{REFERENCES}

[1]. LI, Z., HOU, J., LU, L., TANG, S. \& MA, J. On residents' satisfaction with community health services after health care system reform in Shanghai, China, 2011. BMC Public Health, 2012. Springer, S9.

[2]. OLOMI, G., MBOYA, I. \& MANONGI, R. Patients' level of satisfaction with the health care services received at outpatient departments in Kilimanjaro region. Tanzania. J Patient Care, 2016, Vol. 27, 1-6.

[3]. XESFINGI, S. \& VOZIKIS, A. Patient satisfaction with the healthcare system: assessing the impact of socio-economic and healthcare provision factors. BMC health services research, 2016, Vol. 16, p94.

[4]. OWAIDH, A. O., ATIAH, A. A., ABADI, A. S., ALI, A. M., ABDULlAH, A. M., ABDULLAH, A. A. \& HASSAN, A. M. Patients' satisfaction with health care services in Southern Saudi Arabia. Egypt J Hosp Med, 2018, Vol.72, 3857-3860.

[5]. WHO. What is Quality of Care and why is it important?, n.d.

[6]. TAYLOR, K. Paternalism, participation and partnership - the evolution of patient centeredness in the consultation. Patient education and counseling, 2009, Vol.74, 150-155.

[7]. OCHAN, A. W., AARON, K., ALIYU, S., MOHIUDDIN, M. \& BAMAIYI, P. Patients' satisfaction with Healthcare Services Received in Health Facilities in Bushenyi District of Uganda. International Journal of Science and Healthcare Research, 2018, Vol.3, 76-87. 
[8]. BARRY, C. A., STEVENSON, F. A., BRITTEN, N., BARBER, N. \& BRADLEY, C. P. Giving voice to the lifeworld. More humane, more effective medical care? A qualitative study of doctor-patient communication in general practice. Social science \& medicine, 2001, Vol.53, 487-505.

[9]. OGUNFOWOKAN, O. \& MORA, M. Time, expectation and satisfaction: patients' experience at National Hospital Abuja, Nigeria. African Journal of Primary Health Care \& Family Medicine, 2012, Vol.4.

[10].SHUMBA, C. S., KABALI, K., MIYONGA, J., MUGADU, J., LAKIDI, L., KERCHAN, P. \& TUMWESIGYE, T. Client satisfaction in a faith-based health network: Findings from a survey in Uganda. African health sciences, 2017, Vol.17, 942-953.

[11].KWESIGA, D. A comparative analysis of client satisfaction among people receiving HIV/AIDS care from public and private health facilities in Kabale District. Makerere University, 2010.

[12].UBOS, The National Population and Housing Census 2014 - Area Specific Profile Series Mpigi District. Kampala. UBOS, 2017.

[13].MOHAN, D. R. \& KUMAR, K. S. A study on the satisfaction of patients with reference to hospital services. Int J Bus Econ Manag Res, 2011, Vol.1, 15-25.

[14].DONG, W., ZHANG, Q., YAN, C., FU, W. \& XU, L. Residents' satisfaction with primary medical and health services in Western China. BMC health services research, 2017, Vol.17, 298.

[15].KIGULI, J., EKIRAPA-KIRACHO, E., OKUI, O., MUTEBI, A., MACGREGOR, H. \& PARIYO, G. W. Increasing access to quality health care for the poor: Community perceptions on quality care in Uganda. Patient preference and adherence, 2009, Vol.3, 77.

[16].Ojok, F., Mukasa, S. \& Omona, K. Determinants of Health Service Utilization Among Users of Dokolo Health Centre IV, Dokolo District: A Community Perspective. International Journal of Health, 2021, Vol.8 (1), 17-25.

[17].STAMBOGLIS, N. \& JACOBS, R. Factors Associated with Patient Satisfaction of Community Mental Health Services: A Multilevel Approach. Community mental health journal, 2020, Vol.56, 50-64.

[18].GOBEN, K. W., ABEGAZ, E. S. \& ABDI, S. T. Patient satisfaction and associated factors among psychiatry outpatients of St Paulo's Hospital, Ethiopia. General Psychiatry, 2020, Vol.33.

[19].PILOYA, S. Determinants Of Patient Satisfaction With Health Care Services At Out Patient Department Of Gulu Regional Referral Hospital. International Health Sciences University, 2017.

[20].FUFA, B. D. \& NEGAO, E. B. Satisfaction of Outpatient Service Consumers and Associated Factors Towards the Health Service Given at Jimma Medical Center, South West Ethiopia. Patient Related Outcome Measures, 2019, Vol.10, 347.

[21].NAIR, M., YOSHIDA, S., LAMBRECHTS, T., BOSCHI-PINTO, C., BOSE, K., MASON, E. M. \& MATHAI, M. Facilitators and barriers to quality of care in maternal, newborn and child health: a global situational analysis through metareview. BMJ open, 2014, Vol.4, e004749.

[22].GADALLAH, M., ZAKI, B., RADY, M., ANWER, W. \& SALLAM, I. Patient satisfaction with primary health care services in two districts in Lower and Upper Egypt. EMHJ-Eastern Mediterranean Health Journal, 2003, 9 (3), 422-430.

[23].KADUYU, P. Prevalence and factors associated with client satisfaction with intrapartum care at Mulago Hospital. Makerere University, 2014.

[24].LERNER, J. E. \& ROBLES, G. Perceived barriers and facilitators to health care utilization in the United States for transgender people: A review of recent literature. Journal of Health Care for the Poor and Underserved, 2017, Vol. 28, 127-152. 